\title{
The General Factor of Personality (GFP) as social effectiveness: Review of the literature
}

\author{
Dimitri van der Linden ${ }^{\mathrm{a},}$, , Curtis S. Dunkel ${ }^{\mathrm{b}}$, K.V. Petrides ${ }^{\mathrm{c}}$ \\ ${ }^{a}$ Institute of Psychology, Erasmus University Rotterdam, The Netherlands \\ 'Department of Psychology, Western Illinois University, USA \\ ${ }^{c}$ London Psychometric Laboratory, University College London, United Kingdom
}

a r t i c l e in fo

Article history:

Received 28 January 2016

Received in revised form 6 May 2016

Accepted 7 May 2016

Available online 1 June 2016

\section{Keywords:}

General Factor of Personality

Social effectiveness

Trait emotional intelligence

Evolutionary psychology

Individual differences a b s t r a c t

The General Factor of Personality (GFP) is a higher-order factor causing lower-order personality traits to show consistent correlations in a socially desirable direction. The literature on the GFP reveals that there are various scientific interpretations of this construct. One interpretation is that it is a substantive factor reflecting general social effectiveness and exerting a broad influence on behavior. Another interpretation is that it merely reflects methodological or statistical artifacts and has no further relevance for personality research. We review the empirical literature on the nature of the GFP, its possible links to evolutionary processes, and its relation to other constructs overlapping with social effectiveness. We conclude that the substantive interpretation of the GFP is the most plausible, whereas the notion that it is a psychologically meaningless methodological artifact would be rather difficult to uphold.

(c) 2016 Elsevier Ltd. All rights reserved.

\section{Introduction}

The polymath Francis Galton (1887) implied that even though per- sonality may consist of many different facets, there may also exist a common personality factor that exerts a broad and general influence on behavior. Although a relatively novel idea at the time, Galton did not test it statistically. It was Webb (1915) who conducted one of the first factor-analytic studies on this topic, revealing the presence of a general factor. In the following century, the notion of a General Factor of Personality (henceforward GFP) faded into the background and in- stead several personality models were developed under the assumption of multiple, and conceptually independent dimensions, such as the Big Five or Eysenk's Giant Three. Yet, despite the focus on multiple person- ality dimensions, the general factor continued to resurface in the litera- ture throughout the past decades. For example, in the early lexicon studies on personality, a strong general factor emerged that was labeled social desirability (e.g., Saucier, 1994). Other examples of earlier studies reporting or discussing a general factor in personality are Edwards (1957), and Peabody and Goldberg (1989). Thus, the notion of a general factor in personality was never really denied, it just did not receive much attention because researchers often assumed it may largely re- flect response bias.

\footnotetext{
* Corresponding author at: Institute of Psychology, Erasmus University Rotterdam, P.O. box 9104, 3000 DR Rotterdam, The Netherlands.

E-mail address: vanderlinden@fsw.eur.nl (D. van der Linden).
} 
Currently, however, one hundred years after the original study of Webb (1915), the GFP has received renewed attention with a stream of articles examining its nature (Bäckström, Björklund, \& Larsson, 2009; Chang, Connelly, \& Geeza, 2012; Dunkel \& Van der Linden, 2014; Irwing, 2013; Rushton, Bons,; Hur, 2008; Rushton et al., 2009; Van der Linden, Te Nijenhuis, \& Bakker, 2010). Despite the upsurge in literature on the topic, however, it is clear that no consensus has been established yet regarding what the GFP actually represents. Therefore, we considered it relevant and timely to present a review focusing on the nature of this factor. The key question we wishto examineis wheth- erthe GFP reflects a substantivefactor of socialeffectiveness. According- ly, this is not a fully balanced account of all thescientific opinions on the GFP. Eventhoughwe will address several points of critique and alterna- tive explanations, the main emphasis will be on the possibility that the GFP represents a meaningful construct with implications for theories on individual differences.

\subsection{Basic characteristics of the GFP}

The idea of a GFP reemerged due to observations that personality traits show consistent intercorrelations (Figueredo, Vásquez, Brumbach, \& Schneider, 2004; Musek, 2007; Rushton et al., 2008, 2009) leading to a general factor typically explaining 20 to $60 \%$ of the variance among traits (Van der Linden, Te Nijenhuis et al., 2010). This general factor seems to reflect a continuum, with prosocial behavior at the positive end, and antisocial behavior at the negative end (Figueredo et al., 2004; Rushton \& Irwing, 2011). In terms of the well-

http://dx.doi.org/10.1016/j.paid.2016.05.020

0191-8869/@ 2016 Elsevier Ltd. All rights reserved. 
established Big Five model, higher scores on the GFP, on average, imply higher scores on openness to experience, conscientiousness, extraver- sion, agreeableness, and lower scores on neuroticism.

Although diverging opinions exists on thenature of the GFP, its exis- tence has been convincingly demonstrated in numerous articles. Forex- ample, large meta-analyses have now confirmed the presence of the GFP in self and other reported Big Five measures (Rushton \& Irwing, 2011; Van der Linden, Te Nijenhuis et al., 2010). Also, despite two earlystudies suggestingotherwise(DeVries,2011;Hopwood,Wright,

\& Donnellan, 2011), it is becoming increasingly clear that GFPs extract-ed from various personality measures overlap strongly and meaningful-ly (Davies, Connelly, Ones, \& Birkland, 2015; Dunkel, Reeve, Woodley, \& Van der Linden, 2015; Loehlin, 2012; Rushton \& Irwing, 2011; Van derLinden, Te Nijenhuis, Cremer, Van de Ven, 2011).

Accepting that the GFP is indeed present in personality measures, a subsequent question concerns its interpretation. In the present study, we will address this question and also consider points of critique that can be roughly categorized into i) a measurement artifact view, and ii) a statistical artifact view.

\subsection{The GFP as a social effectiveness factor}

The idea that the GFP reflects social effectiveness is currently the leading substantive interpretation of the construct (Dunkel \& Van der Linden, 2014; Figueredo \& Rushton, 2009; Loehlin, 2012; Rushton et al., 2008, 2009). According to this interpretation, individuals high on the GFP may have the knowledge, skills, and motivation to act in ways that others consider socially desirable, which subsequently increases their chances of achieving socialgoals. Consequently, high-GFP individ- uals may havea higher probability of being selected as mate, co-worker, or leader (Rushton \& Irwing, 2011).

Laband field studies providesupport forthis interpretation. Using a socialnetworkanalysis, high-GFPadolescents werefound to berated as more popularand likeable by their classmates (VanderLinden, Scholte, Cillessen, teNijenhuis \& Segers, 2010). High-GFP individuals also seem to have an advantage in personnel selection procedures; for example, they are more likely to be hired in leadership positions in the Dutch army (Van der Linden, Te Nijenhuis, Cremer, Van de Ven \& Van der Heijden-Lek, 2014), and tend to obtain higher overall scores in assess- ment centers (Van der Linden, Bakker \& Serlie, 2011). High-GFP em- ployees obtain higher performance ratings by their supervisors too (Van der Linden, Te Nijenhuis et al., 2010). Of course, given the subjec- tive nature of supervisor ratings, it remains a question whether high- GFP employees perform better objectively or are only rated as such. Yet, at least one published study found that high GFP scores were relat- ed to higher supervisor ratings as well as better sales performance, mea- sured by thenumber of new customers acquired (Sitser, VanderLinden,

\& Born, 2013).

Also in accordance with the social effectiveness interpretation are labstudies confirming that high-GFP scores arelinked tohigher perfor- mance on ability tests of social knowledge and skills (Dunkel, Summerville, Yockey, Reeve, Stolmeier, \& Kesserling, 2014; Van der Linden, Oostrom, Born, Van der Molen and Serlie, 2014). Importantly, the GFP shows strong associations with other established measures of social effectiveness and emotional intelligence, more of which later.

It is imperative to state that the interpretation of the GFP as social ef- fectiveness does not imply that a single factor suffices to describe an individual's full personality. It is obvious that individuals can and do dif- fer on multiple dimensions; for instance, being hard-working (conscientiousness) does not inevitably imply that one is also friendly (agreeableness). Thus, the GFP is not a replacement for more nuanced models of personality because it is unlikely that character can be re- duced to a single dimension. Rather, the GFP implies that there are gen- eral tendencies or mechanisms pushing multiple traits towards a broad dimension of socialdesirability. This idea, if correct, provides usefuland important insights into the structure of personality. 
Considering the GFP as substantive raises a question about its ori- gins. One possible answer to this question is that the GFP has been shaped by evolutionary selective pressures towards socially desirablebehavior (Figueredo \& Rushton, 2009; Figueredo et al., 2004). Figueredoand co-workers were among the first to test this idea systematically. In2004, Figueredo et al. argued that a GFP exists that may be considered asone of the indicators of a so-called Life History (LH) strategy. LH theoryis a mid-level evolutionary account of differences in reproductive strat-egies (Figueredo et al, 2004). The theory makes predictions regarding reproduction based on energy allocation. For example, an individualmay direct energy away from reproduction (e.g., finding mates) to so-matic effort (e.g., maintaining health). The basic premise of LH theoryis that a continuum exists of fast versus slow $(\mathrm{LH})$ reproductive strate-gies. A fast strategy is characterized by the production of many off-spring, but providing relatively little parental care. Such a strategy isassumed to have evolved in environments where there is high mortalitydue to unpredictable dangers (e.g., predators and pathogens; Figueredo, Woodley of Menie, \& Jacobs, 2015). At the other end, a slow LHstrategyis characterized by fewer offspring, but providing a lot of parental care.A slow LH strategy would have evolved, and fit better in environments where the limiting factor for population growth is the amount of avail-able resources and where dangers are more predictable.

Originally developed to explain differences in reproductive behavior betweenspecies, LHtheory can also be applied toexplainindividual dif-ferences within species. This idea is relevant for psychological research on individual differences because it has been argued and shown thatLH strategy in humans is related to a wide range of psychological traits,such as sexual attitudes and behavior, health, motivation, and also per- sonality (Figueredo et al., 2004; Rushton, 1985), which is where the GFP becomes relevant. Figueredo and colleagues have made the case that a slow LH strategy requires close cooperation between members of a group as well as between parents in order to increase the survivability of the offspring. Figueredo and Rushton (2009) argued that "...Weshould therefore expect life history evolution to favor the evolution of the GFP, because the conditions favoring slow life history strategy are those fa-voring the cooperative sociality indicative of the GFP." (p. 556).

Although the literature shows that various selective evolutionary mechanisms may have had their effect on personality (e.g., Penke, Miller, \& Denissen, 2007), several researchers argued that for a broaderpersonality factor such as the GFP, directional evolutionary selectionmay be a viable possibility (Figueredo et al., 2015; Rushton et al.,2008). High social effectiveness would be associated with positive socialoutcomes that had relatively strong adaptive advantages during humandevelopment. Specifically, as humans are a social species by nature, in- dividuals knowing how to deal with others, how to get their approval, and how to socially maneuver in order to attain goals may have had higher inclusive fitness, on average (Figueredo \& Rushton, 2009). As fur-ther discussed below, several genetic and behavioral studies support forthis idea.

\subsection{The genetic evidence}

Based on twin data, it is now well-established that the GFP has a ge- netic component of approximately $50 \%$ (Figueredo et al., 2004 ; Loehlin \& Martin, 2011; Rushton et al., 2008; Veselka et al., 2009). A distinction can be made between additive and non-additive genetic effects (Falconer, 1989). Non-additive genetic variance is often assumed to in- dicate that a trait has been under the influence of natural selection, be- cause selection tends to deplete additive genetic variance faster than non-additive genetic variance (Falconer, 1989). Regarding this, the GFP has been shown tohave significant non-additive heritability. Bell, Woodley, Schermer, and Vernon (2012) as well as Rushton et al. (2008) reported 50\% nonadditive genetic variance. However, there are also studies that could not replicate the presence of strong genetic 
dominance effects in the GFP (Loehlin \& Martin, 2011). Nevertheless, as the majority of published studies do indicate the presence of non-addi-tive genetic variance in the GFP, it is reasonable to believe that the con-struct has been under recent evolutionary selective pressure (Figueredo

\& Rushton, 2009).

In genetic research, inbreeding effects are considered to indicate di- rectional selection (Falconer, 1989) and can, therefore, also be used to test hypotheses about selection effects in the GFP. Inbreeding refers to offspring sired by (distant) relatives. Such inbreeding can have detri- mental effects on various traits because it may cause deleterious reces- sive mutations to cluster in an individual, which is often referred to as 'mutationload'.

Verweij et al. (2012) conducted a study on inbreeding by testing so- called runs of homozygosity (RoH) in a sample of 5530 participants.RoHs indicate the amount of identical stretches of DNA that can be ob-served in the offspring of close, but also fairly distant, relatives (i.e., in- dividuals sharing a distant ancestor). Verweij et al. found that a highernumber of RoHs (more inbreeding) was associated with lower scores on the GFP. These findings were interpreted as evidencethat the sociallydesirable end of personality scales (i.e., the GFP) has been under direc-tional evolutionary selection (Verweij et al., 2012).

Another interesting piece of genetic evidence linked to the GFP comes from the recent study by Pettersson, Larsson, and Lichtenstein (2015). Using a sample of 3,475,112Swedish adults they found a gener- al genetic factor relating to a wide range of psychological and social problems, suchas depression, ADHD, alcoholand drugabuse, and a ten- dency towards criminal behavior. They argued that a set of pleiotropic genes influence all these behaviors. Pettersson et al. (2015) also explic- itly acknowledged that their general genetic factor is likely related to the GFP, and mentioned the possibility that it could be interpreted as a social effectiveness factor ("....an evolved tendency favoring more co- operate and stable personalities" Pettersson et al., 2015,p.4).

More generally, current genetic evidence points to the direction of a GFP that has a (non-additive) heritable component, which suggests that it offered advantages to individuals displaying a general tendency to- wards socially desirablebehavior. The detrimental effects of mutation- load, as apparent ininbreeding studies, furtherindicates that evolution- ary selectiveforces would havefavored thosewithhigherscores onthis trait.

\subsection{The behavioral evidence}

An indirect approach to examining evolutionary accounts of the GFP involves testing specific behavioral predictions arising from it. For ex-ample, Figueredo et al. (2004) used the Mid-Life Development in the US (MIDUS) study to construct a wide range of LH indicators (e.g., pa- rental support and relationships, health, delinquency, and social status)all of which showed overlap with the GFP. In subsequent studies, the status of the GFP as a LH strategy indicator was further consolidated (Dunkel \& Decker, 2010; Dunkel, Kim, \& Papini, 2012; Olderbak,Gladden, Wolf, \& Figueredo, 2014).

The GFP-LH strategy relationship has been supported by self-report studies, but also studies using other ratings or objective outcomes (e.g., Dunkel, Nedelec, \& Van der Linden, 2015), thus it cannot be attrib- 


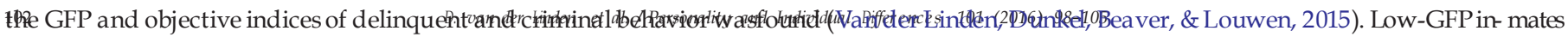
were significantly younger when they were arrested for thefirst time, were more likely to have been arrested multiple times intheir life, and showed more behavioral problems (as rated by the war-dens) during their detention. Table 1 shows the correlations betweenvarious measures of delinquency and adjustments to prison life and the GFP extracted from the California Psychological Inventory (CPI).

In so far as the GFP stems from evolutionary selective forces, one specific prediction would be that it is universal and can be found among all cultures and societies. Cross-cultural research indicates that this is indeed the case. De Raad et al. (2010) conducted a large-scale per-sonality study with 14 trait taxonomies in 12 languages. One of theirconclusions was that a general factor, i.e., the GFP, was consistentlyfound in all languages.

Regarding the expression of the GFP in different cultures, Dunkel (2013) compared GFPs in a US and a Japanese sample and found thatthe GFP is related to enculturation, which refers to the extent to whichone adopts cultural norms. This relation between manifestation of theGFP and cultural norms, was further supported by the study of He and van de Vijver (2013) who compared individuals with various culturalbackgrounds in the Netherlands. From the substantive interpretationof the GFP, it may not be surprising that the general factor is influencedby cultural norms, since, in order to be socially effective one often has toadapt to the general rules of social interaction of one's culture.

All in all, the genetic and behavioral datapresented above are in line with the interpretation of the GFP as broad and universal personality factor that has been shaped by evolutionary selective pressure towards socially desirable behavior.

\section{GFP criticisms}

Despite accumulating evidence supporting the theoretical and prac- tical relevance of the GFP, the construct is certainly not beyond criticism. Some have even argued for a full stop on GFP research (De Vries, 2011). We do not agree with this point of view because it is obvious that many questions regarding this construct are still open. In order to provide a sense of the alternativeinterpretations available, wesummarizeseveral key studies that have argued against the relevance of the GFP for per- sonality research. Roughly, two main categories of criticisms can be identified, one that considers the GFP as mere social desirability bias and another that considers it as a statistical artifact.

\subsection{Social desirability bias}

Among the first studies arguing that theGFP reflects socialdesirabil- ity bias were thoseof Bäckström and colleagues (Bäckström, Björklund,

Table 1

Bivariate correlations between theGFP and delinquent behavior and previous psycholog- ical and social problems $(N=1345)$.

Extracted from: Van der Linden, Dunkel, Beaver, \& Louwen, 2014.

uted tomethod biases. For example, based ona sampleof approximate-

GFP ly 400 families, Van der Linden, Figueredo, De Leeuw, Scholte, and Engels (2012), showed that higherGFP scores from parental self-reports were associated with higher ratings of parental support as independent- ly provided by their children. Higher levels of parental support would be characteristic for families with a relatively slow LH strategy.

Another widely used indicator in LH theory is the tendency towards delinquent behavior (Dunkel, Mathes, \& Beaver, 2013; Rushton, 1985). Individuals with a fast LH strategy are presumed to have a tendency for rule-breaking and impulsive behavior as a way of competing with other fast-LH strategists for access to resources and potential mates. Using large sample of inmates in theUS, a direct relationship between 
Age at first arrest

Total number of arrests

Interpersonal adjustment in prison

Work adjustment in prison

School problems

Interpersonal relations problems

Childhood maladjustment and deviance

Adult maladjustment and deviance

Social marginality

Socioeconomic status

General delinquency factor

Note:

II $p$ b .001.
D. van der Linden et al. / P0.21 fatality and Individual Differences 101 (2016) 98-105

10 $-0.13^{\amalg}$

0.14

$-0.34^{\square[}$

$-0.23^{\amalg}$

$-0.22$

$-0.22^{\mathbb{W}}$

$-0.33^{\text {II }}$

$0.38^{\square}$
$-0.28^{\square}$ 

\& Larsson, 2014; Bäckström etal.,2009) who showed that when items are reformulated in a way that does not include a socially desirable di- rection, the shared variance of personality traits (i.e., the GFP) is reduced (e.g., "Gets upset easily" was reformulated into "Sometimes reacts strongly to things that happen"). These studies have often been cited as evidenceagainst a substantive GFP.

At a closer look, however, the interpretation of these findings may not be so straightforward. First, even after reformulating the items,Bäckström et al. (2009) found that a substantial proportion of sharedvariance remained present. Second, and more importantly, it is not clear to what extent one can take away social desirability from itemswithout changing their actual content. One may no longer be measuringthe same behavior. Third, if the GFP reflects social effectiveness, then weshould expect it to be small when extracted from items with reduced social desirability variance.

Another study showed that participants scoring high on the GFP also tend to endorse personality items formulated in a socially positive way (Pettersson, Turkheimer, Horn, \& Menatti, 2012). Subsequently, it was argued that the GFP mainly indicates evaluative aspects of personality measures rather than the genuine behavior of the participants, which is anotherway of stating that theGFP reflects mereresponsebias. A lim- itation of this study, however, is that it did not include criterion mea- sures (e.g., actual behavior and performance). Thus, it could not be directly established whether the tendency to endorse evaluative items in high-GFP individuals reflected substanceor artifact. Infact, a subse- quent study by Bäckström et al. (2014) suggests that the specific socially desirable factor in personality measures indeed relates to job perfor- mance. This is a finding that contradicts the purely artifactual interpre- tation of the GFP.

One of the largest studies in this area was conducted by Chang et al. (2012), who used meta-analytic data and structural equation modeling (SEM) in order to test the artifact account of the GFP. They analyzed the trait intercorrelations (e.g. between conscientiousness and extraver- sion) based on different raters and constructed a complex model based on self-and other ratings of personality. They found that when in- tercorrelations between the Big Five dimensions were based on a com- bination of self- and other ratings, the GFP diminished substantially. Hence, they concluded that no substantive GFP exists beyond method effects.

Three key issues regarding this conclusion are worth discussing. First, focusing on trait intercorrelations among raters instead of directly comparing factors obtained by self- and other ratings, may lead to un- derestimation of the importance of higher-order factors. Specifically, within traits (e.g., the Big Five), the correlations among raters tend to be moderate, varying from 0.30 to 0.60 (Connelly \& Ones, 2010). Now, if there is already such a relatively modest overlap between raters on one particular trait, which either implies a certain amount of measure- ment error or rater-unique information, then it can be expected that in- tercorrelations between traits (say, between conscientiousness and emotional stability) will be likewise artificially attenuated to an even greater extent. This is similar to correlations between variables becom- ing attenuated due to unreliability.

A second issue is that the notion that the GFP completely disappears when considering multiple-rater data is not in accordance with studies showing that GFPs based on self- and other ratings do indeed signifi- cantly overlap (e.g., Dunkel, Van der Linden, Brown, \& Mathes, 2016; Rushton et al., 2009; Van der Linden, Figueredo, et al., 2012, Van der Linden, Tsaousis, et al., 2012). Theoretically, a complete lack of conver- gence among raters would not make much sense anyhow, because, if it is established that raters converge on lower-order traits, such as the BigFive, then it is rather unlikely that there will be a lack of convergence at the level of the GFP, since the latter is present in each of the lower- order personality dimensions (see Fig. 1).

Third, a noteworthy line of research that contradicts the foregoing critique on the GFP shows that the social desirability component in personality measures (which is mostly the GFP) appears to be substantive 


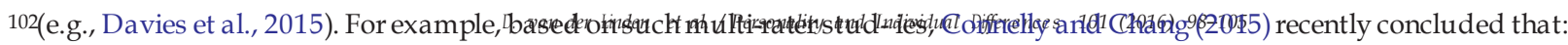

"...SD [Social desirability] scales are strongly affected by substantive traits, particularly Conscientiousness, Emotional Stability, and Agree- ableness. SD scales are more strongly influenced by these traits thanby self-report response styles" (p. 11).

This idea that personality traits fall along an axis of socially undesir- ableversus desirablebehavior, which largely reflects consensually valid trait variance, rather than bias, has also been acknowledge in studies based on the HEXACO personality model (e.g. De Vries, Zettler, \& Hilbig, 2014). The existence of a general substantive dimension of so- cially desirable behavior as reported in these studies, accords well with our conceptualization of theGFP. Alsoin accordancearethe recent findings of Dunkel et al. (2016) who showed that even though the GFP may reflect some level of bias or error (as do all measures in social sci- ence), a substantial proportion of its variance can be ascribed to social effectiveness.

\subsection{The statistical artifact view}

Statistical artifact interpretations differ from social desirability bias in- terpretations in the sense that they do not necessarily provide specific alternative explanations for the GFP, but rather purport that it is irrelevant (Revelle \& Wilt, 2013). Different variations of this critique exist in the literature. Earlier studies suggested that factors extracted from different personality measures differ widely from each other and do not even re- flect the same construct (De Vries, 2011; Hopwood et al., 2011). This ar- gument refers to lack of measurement invariance. Accordingly, De Vries (2011) found that general factors extracted from Big Five and HEXACO measures did not overlap. Similarly, Hopwood et al. (2011) reported low intercorrelations among GFPs extracted from eight different person- ality measures. After these initial studies, however, research demonstrat- ed that GFPs extracted from different measures overlap substantially (Davies et al., 2015; Dunkel et al., 2015; Loehlin \& Martin, 2011; Loehlin, 2012; Rushton \& Irwing, 2011; Van der Linden, Te Nijenhuis, et al., 2011). Moreover, Loehlin (2012) reanalyzed the Hopwood et al. (2011) data by comparing the first unrotated (general) factors instead of complex hierarchical factor analyses in each survey, and concluded that the GFPsin their dataset overlapped substantially and meaningfully. Overall, theaverage, uncorrected,overla pbetweenGFPs is approximately $r=0.60$, which is rather similar to the overlap in several other psycholog- ical constructs (e.g., the Big Five; Connelly \& Ones, 2010). Using confirma- tory factor analysis with statistical corrections for artifacts, like sampling error and unreliability, Rushton and Irwing (2011) reported that the over-lap between GFPs extracted from various instruments appears to be ap- proaching unity.

Another point of critique that has been raised is that it is statistically possible to extract a first unrotated factor of substantial size from any set of (personality) measures, without it being a truly general factor. Using simulated data, Revelle and Wilt (2013) showed that a relatively large first unrotated factor could be extracted from Big Five measures, even though the average intercorrelations among these measures was low. As Revelle and Wilt (2013) proved, there is no doubt that this mathematical possibility exists. However, this possibility is at variance with a host of evidence showing that most lower-order personality traits do, in fact, loadontheGFP, whichsuggests that thefirst unrotated factor in personality measures is a good operationalization of the GFP (Rushton \& Irwing, 2011; Van der Linden, Scholte, et al., 2010).

The last statistical critique we address purports that higher-order per- sonality factors (above the Big Five or Big Six level) are caused merely by correlated personality facets (Ashton, Lee, Goldberg, \& De Vries, 2009). This is referred to as the so-called 'blended variable' or 'blended facet' models. This argument posits that intercorrelations between facets are captured in the mid-level factors (i.e., the Big Five or the HEXACO) as 


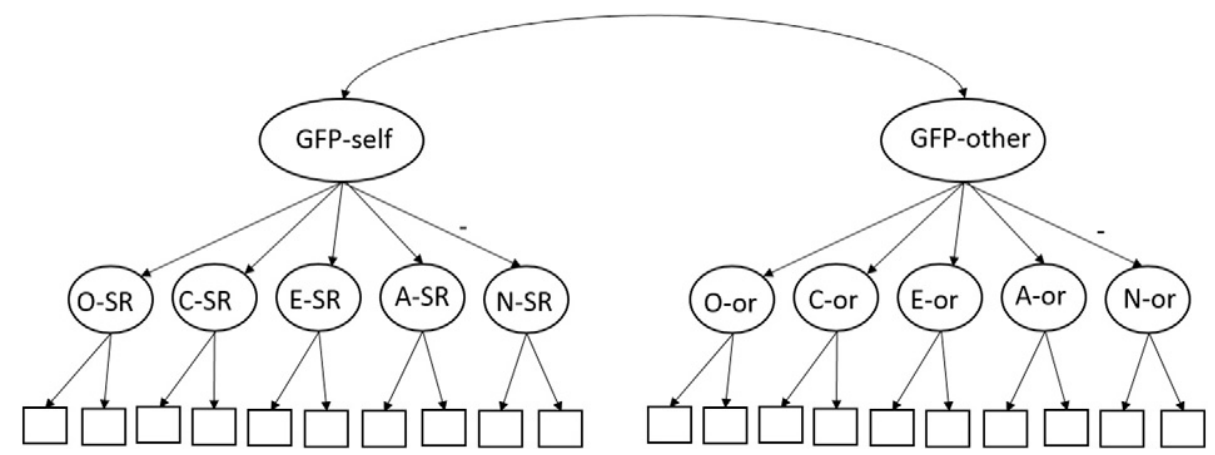

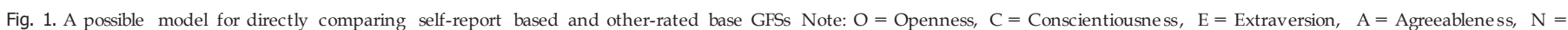
Neuroticism, SR = self-rated, or = Other-rated (squares represented lower level facets or items).

these are extracted from the facets. Consequently, it is suggested that when trying to extract higher-order factors one should control for facet intercorrelations. One assumption of the blended variable model is that there are no substantive higher-order factors above the Big Five or HEXACO. Thus, this model denies the existence of not only the GFP, but also of other higher-order personality constructs, such as Stability and Plasticity (DeYoung, 2006; or Alpha and Beta; Digman, 1997). These two higher-order factors consist of conscientiousness, agreeableness, and emotional stability, on the one hand, and openness and extraversion, on the other.

In our view, and those of several other researchers (e.g. Irwing, 2013; Veselka, Just, Jang, Johnson, \& Vernon, 2012), theblended variable model has several limitations that are still open to discussion. One lim-itation is that by controlling for correlated facets or by allowing crossloadings between facets and criterion variables, one also controls for true overlap between personality factors, such as the Big Five or the

HEXACO dimensions, which artificially diminishes theimportance of any higher-order factors. Veselka et al. (2012) concluded that "...this logic ensures that a GFP will never be recognized even if it exists" (p. 262). In general, blended variable models as well as models assuming a general factor, reflect a situation in which personality dimensions are not independent of each other, but show genuine and meaningfulinter- correlations.

The difference lies in how one chooses to model these cor- relations statistically. We agree with Jensen (1987) that several equivalent statistical

solutions are possiblethat reproduce the original correlations between trait or ability measures, but that some solutions make more sense,

theoretically, than others and some solutions may even be misleading. So, in the end, which model is the most plausible one has to be decided on theoretical arguments. In accordance with this, in the current review we wish to make the case that general social effectiveness (or emotional or social intelligence) functions as a General Factor of Personality and is a plausible explanation for the intercorrela-

tions between traits.

\section{The GFP and emotional intelligence}

If theGFP is indeed a socialeffectiveness factor, it would beexpected to overlap with measures of emotional intelligence (EI). Broadly de- fined, EI concerns differences in the extent and manner in which people experience, express, and utilize affect-laden information. Since Petrides and Furnham (2000), the field has split into the two constructs of ability EI and trait EI. The former is defined as the "ability to perceive accurate- ly, appraise, and express emotion; the ability to access and/or generate feelings when they facilitate thought; the ability to understand emo- tions and emotional knowledge; and the ability to regulate emotions to promote emotional and intellectual growth" (Mayer \& Salovey, 1997; p. 10) and ought to be measured via tests of maximum perfor- mance. Trait EI, on the other hand, is defined as a constellation of 
emotional perceptions located at the lower levels of personality hierar- chies (Petrides, Pita, \& Kokkinaki, 2007) and is measured via question- naires and rating scales.

Perhaps to different degrees, both ability and trait EI imply that indi- viduals with high scores know how to regulate their emotions, what constitutes socially desirable behavior, and how to act in order to attaingoals. However, given the extensive conceptual and methodological dif-ferences between the two EI constructs, it is appropriate to examinetheir relationships with the GFP in separate subsections.

\subsection{Ability EI and the GFP}

There are comparatively few studies on the link between ability EI and the GFP, both because ability EI is a smaller research domain than trait EI, but also because, conceptually, the construct is firmly situated within the human abilities hierarchy. Nonetheless, existing evidence shows that ability EI is positively and significantly related to the GFP. In what was perhaps the first study on the topic, McIntyre (2010) re- ported lowto-moderate positive loadings from the Mayer-Salovey-Ca- ruso-Emotional-Intelligence-Test (MSCEIT) factors on the GFP. These loadings were somewhat stronger for females than males. Van der Linden et al. (2016) in a series of meta-analyses, also found overlap be- tweenability EIand the GFP. More specifically, principalaxis factoring (PFA) performed onanability EI-Big Fivematrix, arising from 47 studies with a total sample size of 10,258 , revealed a moderate loading of 0.22 from ability El on the first unrotated factor. Structuralequationmodel- ing (SEM) applied to the same meta-analytic data set showed that abil- ity EI correlated $r=0.27$ with a GFP extracted from the Big Five personality dimensions (see Fig. 2).

Overall, it seems clear that there is modest overlap between ability EI and the GFP. Given the ambiguities in the scoring methodology of the MSCEIT, thesource of this overlap is not easily ascertained.Forexample, it has been argued that MSCEIT scores may be confounded with vocab-ulary size, conformity to social norms, theoretical knowledge about emotions, stereotypical judgments, or some unknown combination of these factors. This makes it difficult to establish which of the factors play a role in the construct's overlap with the GFP (Petrides, 2009). In- deed, such methodological and interpretational challenges facing the construct of ability EI (see also Fiori et al., 2014) are perhaps one reason why most research in the field is conducted within the trait EI domain.

\subsection{Trait EI and the GFP}

Comparatively more studies have been performed on the relation- ship between the GFP and trait EI. Rushton et al. (2009; Study 3) ana- lyzed a twin dataset including the Big Five, four humor styles, and the four trait EI factors based on the short form of the Trait Emotional Intel- ligence Questionnaire(TEIQue-SF). They randomly divided thedata set 
into two halves and separately extracted GFPs in the two samples, which yielded nearly identical results. In the first sample, the GFPaccounted for $33 \%$ of the total variance in the matrix, including strong loadings on all four trait EI factors: Well-being (0.84), Self-Control(0.70), Emotionality (0.77), and Sociability (0.63), while in the second sample it accounted for $31 \%$ of the total variance, with the following trait EI factor loadings: Wellbeing (0.84), Self-Control (0.62), Emotion-ality (0.73), and Sociability (0.69).

In a similar design using two different twin samples, Veselka et al. (2009) extracted GFPs from the Big Five and the 15 trait EI facets based on the full form of the TEIQue. In the first sample, the GFP accounted for $38.6 \%$ of the total variance in the matrix. The average loading for the 15 trait EI facets was 0.64 , with none falling below

0.30. Inthesecond sample, theGFP accounted for $35.2 \%$ of thetotalvar- iance in the matrix. The average loading for the 15 trait EI facets was 0.62 , with none falling below 0.30. In a different study based on a similar design, Veselka et al. (2009) extracted a GFP from a combined dataset of the six HEXACO and the fourtrait EI(TEIQue-SF) factors, separately in two randomly-split twinsamples. Inthefirst sample, theGFP accounted for $32.8 \%$ of the totalvariance in the matrix, with the following loadings on trait EI: Well-being (0.79), Self-Control (0.75), Emotionality (0.67), and Sociability (0.77), while in the second sample, it accounted for $32.9 \%$ of the total variance, with the following loadings: Well-being (0.77), Self-Control (0.76), Emotionality (0.67), and Sociability (0.73).

Van der Linden, Tsaousis, and Petrides (2012) extracted GFPs from Big Five and Giant Three factor, facet, and parcel scores, which they subsequently correlated with global trait EI(TEIQue) scores. The average correlation was $r=0.72$. These correlations were barely affected by partialing out social desirability scale scores. Last, Pérez-González and Sanchez-Ruiz (2014) extracted a GFP from Big Five data, which correlat-ed at $r=0.69$ with global trait EI (TEIQue). In addition, they extracted aGFP from a combined Big Five - trait EI dataset, on which the average facet loading was 0.58. In their exhaustive meta-analysis, van derLinden et al. (2016) analyzed data from 95 samples with a total samplesize of 30,198. They extracted a GFP via PFA, which explained $27 \%$ of the total variance in a matrix comprising the Big Five and global trait EI. At 0.69 , global trait EI had the highest loading on this GFP. A subsequent CFA showed that the GFP correlated at $r=0.86$ with global trait EI (see Fig. 2).

Taken together, the findings above indicate that there is a strong positive relationship between the GFP and trait EI. This, of course, is fully in line with the hypothesis that emotional intelligence is best con-ceptualized as a personality trait, rather than a cognitive ability (Petrides, 2010). But what is the nature of this relationship? Recent re-search has revealed that many of the genes that are responsible for indi- vidual differences in the Big Five are also implicated in individualdifferences in trait EI (Van der Linden et al., 2016; Vernon, Vanessa, Villani, Schermer, \& Petrides, 2008). Psychologically, the large overlap between the GFP and trait EI stems from the fact that while personalityspans a very wide remit, including attitudes intentions, and motives, its main component is emotion. It follows that trait EI, which is 


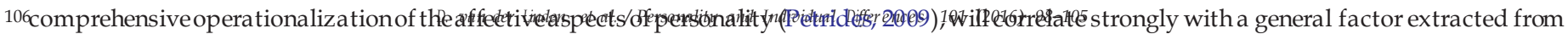
a sampling domain, such as that of the Big Five, that deals mainly, if haphazardly, with emotions. The contribution of trait EI is that it cen- tralizes the emotion-related variance that is scattered among the five, supposedly orthogonal, higher-order personality dimensions and aug- ments it by incorporating significant additional variance as reflected in its compelling evidence of incremental validity (Andrei, Siegling, Aloe, Baldaro, \& Petrides, 2015).

\section{Synopsis and discussion}

The GFP has elicited widely diverging scientific views. Somescholars are prepared to entertain the hypothesis that it reflects a viable con-struct of social effectiveness, while others outright reject the notion of a meaningful general factor in personality measures. In our view, thelatter position is difficult to maintain in light of the large body of evi-dence supporting the validity and relevance of this factor. In addition, the literature shows that compared to other constructs in social science, the GFP yields robust results. Recently, in the community of social sci-ence research there has been much emphasis on the reproducibility of findings (Open Science Collaboration, 2012). Several established find-ings in psychology are now being questioned because they can onlybe replicated under very specific and restricted conditions (e.g., Carter

\& McCullough, 2014). This is not so for the GFP. First, multiple studies and meta-analyses have clearly confirmed the presence of a sizable proportion of shared variancein personality measures (e.g. Figueredoetal., 2004; Musek, 2007; Rushton \& Irwing, 2011; Van der Linden Te Nijenhuis et al., 2010). Second, each individual researcher in possession of one or more personality datasets can easily replicate this key finding with their own data. It can also easily be replicated that this GFP in per- sonality measures significantly relates to other meaningful criteria mea- sures, such as (other-rated or objective) job performance or social behavior. Thus, the GFP and its relevance is consistent and open for ev- eryone to test. In contrast, forcing this general factor to 'disappear' often requires a very specific and complex set of data analytic steps that in- volve questionable assumptions such as controlling for correlations be- tween facets or traits (Ashton et al., 2009).

Additional support for the GFP as a meaningful construct can be found in the literatures that have examined factors that have been operationalized along the same lines, but labeled differently, such as the 'ideal employee factor' (Klehe et al., 2012), 'impression management' (De Vries et al., 2014), and a 'cultural filter influencing how one's per- sonality manifests itself in society' (He \& van de Vijver, 2013). This body of research concludes that the factors under consideration are sub- stantive and their influence is not restricted to limited (e.g., filling out questionnaires) or high-stake (e.g., selection interviews) situations, but is rather consistent over time and contexts. As such, the underlying factor that causes personality traits to correlate (whatever label one uses for it) would be an important part of what defines someone as a person and how he or she deals with daily social demands.

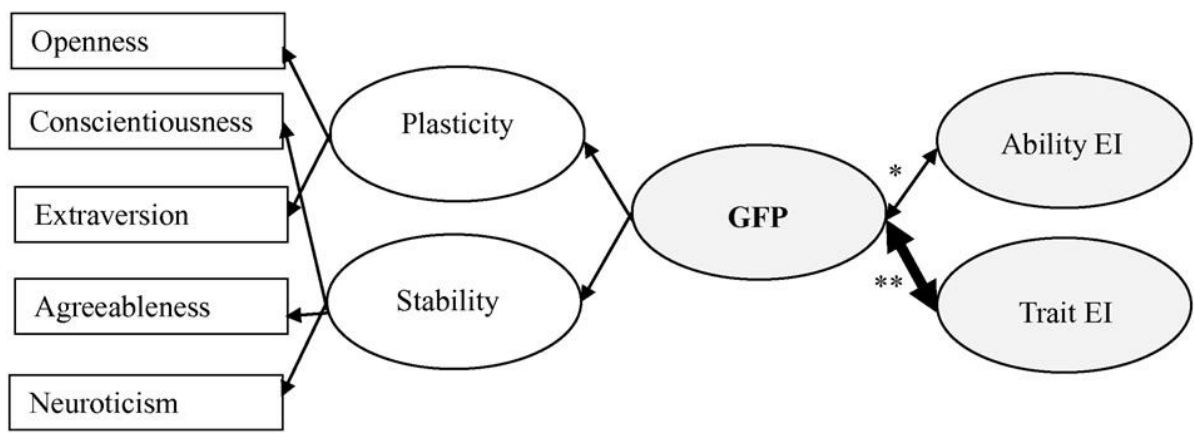

Fig. 2. The GFP and Ability and Trait EI Note: ${ }^{*}$ moderate overlap (somewhere between $.20-.30$ ), ${ }^{* *}$ overlap approaching unity (N.85). 
With respect to the future of GFP research, one possibility is that ac- cumulating evidence will eventually lead to consensus. Whether such a consensus will be reached, and within what timeframe is difficult to predict. For a comparison, we may look at research on the well-known $g$ factor of mental ability. This construct also started with many different views and debates, which still persist today after more than hundred years of systematic research (Jensen, 1998). Another similarity between current research on the GFP and earlier research on the $g$ factor is that many of the arguments that had initially been used in the $g$ factordebate seem to have been recycled in the context of the GFP debate (e.g., unde- fined, abstract, statistical artifact, no contribution beyond lower-order measures).

Irrespective of one's current view of the GFP, we believe that the available empirical data have consequences for future research on indi- vidual differences. For instance, if one maintains that the GFP is a fully artefactual or psychologically 'empty' construct, then this may requireto control for this factor in personality research (see Dunkel, De Baca,Woodley, \& Fernandes (2014) as opposed to Major, Johnson, and Deary (2014)). Yet, doing so will likely affect the outcomes of previously published research. Specifically, there are many examples in which a criterion of interest such as job performance, psychopathology, orhealth behaviors are correlated to the Big Five in a pattern of $\mathrm{O}+, \mathrm{C}+, \mathrm{E}+, \mathrm{A}+$ and $\mathrm{N}-$. What would this mean? That the unique characteris-tics of the Big Five each, and via independent pathways, relate to the cri-terion of interest? Often, a simple test will reveal that it is, in fact, theshared variance of the Big Five (or other personality traits) that is re-sponsible for underlying relationship and that removing this variancefrom the equation has dramatic effects on the results. A prime exampleis Study 2 in Van der Linden Te Nijenhuis et al. (2010), who found that the direct correlations between the Big Five and job performance were 0.28, 0.19, 0.10, 0.05, and 0.14, for O, C, E, A, and Emotional Stability(ES), respectively. After controlling for the GFP, however, these correla-tions dropped to $0.15,0.05,-0.03,-0.12$, and -0.03 , for $\mathrm{O}, \mathrm{C}$, E, A, andES, respectively.

We believe that the present review of the literature has demon- strated that further research on the GFP may well be valuable. Inter-esting possibilities for such research involve testing criteria frommultiple domains. An example of such a potentially relevant con-struct in another domain, namely psychopathology, is the so-called $p$ factor. Caspi et al. (2014) and Hengartner et al. (2014) published studies advocating a hierarchical structure of psychopathological traits, including a general factor at the apex. Conceptually, this $p$ fac-tor of psychopathological personality appears to occupy the negativepole of the GFP as had been previously proposed by Rushton and Irwing (2011).

All in all, in our view, GFP research has yielded significant new insights into the structure of personality, the possible evolutionary origin of individual differences in personality, and how general mechanisms or tendencies towards social effectiveness (e.g., EI) may fit within personality factor space. The notion of the GFP has the potential to yield new and testable hypotheses that are not im-mediately obvious from the perspective of other theoretical frame-works. Despite that certain theoretical aspects of the construct may be currently unclear or require further empirical scrutiny, we believe there are sufficient indications that the GFP is a substantive know- what-to-do-in-socialsituations factor that relates to how one candeal with social demands and thus improve the odds of achieving subjectively important life goals.

\section{References}

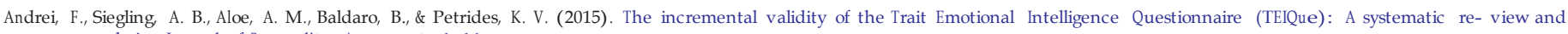
meta-analysis. Journal of Personality Assessment, 1-16.

Ashton, M. C., Lee, K., Goldberg, L. R., \& de Vries, R. E. (2009). Higher-order factors of per- sonality: Do they exist? Personality and Social Psychology Review, 13, 79-91. 


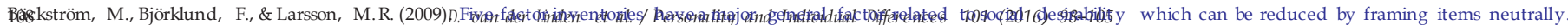
Journal of Research in Personality, 43, 335-344.

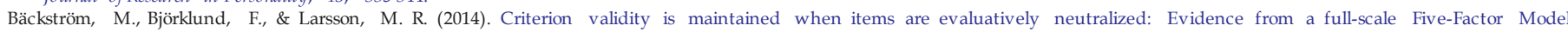
Inventory. European Journal of Personality, 28, 620-633.

Bell, E., Woodley, M.A., Schermer, J. A., \&Vernon, P. A. (2012). Politics and the general fac- tor of personality. Personality and Individual Differences, 53, 546-551.

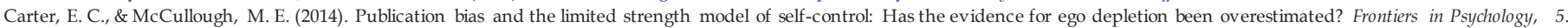
http://dx.doi.org/10.3389/fpsyg.2014.00823.

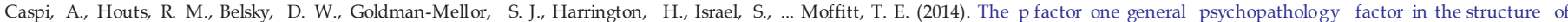
psychiatric disorders? Clinical Psychological Science, 2, 119-137.

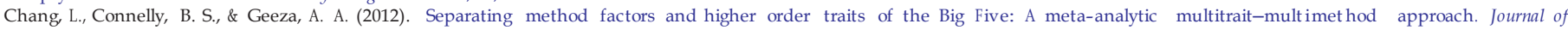
Personality and Social Psychology, 102, 408-426.

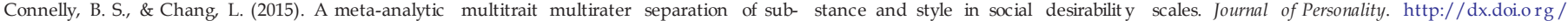
10.1111/jopy.12161 (Online first).

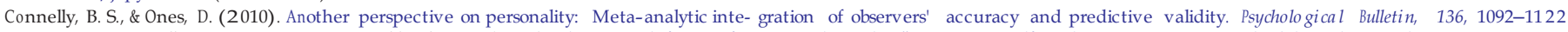

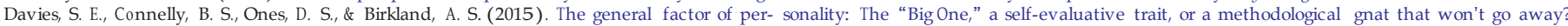
Personality and Individual Differences, 81, 13-22

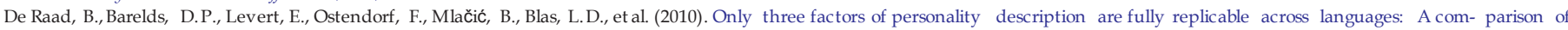
14 trait taxonomies. Journal of Personality and Social Psychology, 98, 160-173.

De Vries, R. (2011). No evidence for a general factor of personality in the HEXACO person- ality inventory. Journal of Research in Personality, 45, 229-232

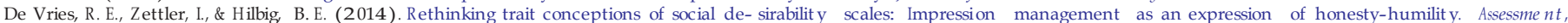
21, 286-299.

DeYoung, C. G. (2006). Higher-order factors of the big five in a multi-informant sample. Journal of Personality and Social Psychology, 91, 1138-1151.

Digman, J. M. (1997). Higher-order factors of the Big Five. Journal of Personality and Social Psychology, 73, 1246-1256.

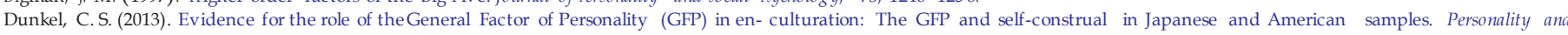
Individual Differences, 55, 417-421.

Dunkel, C.S., \& Decker, M. (2010). Convergent validity of measures of life-history strategy. Personality and Individual Differences, 48,681-684.

Dunkel, C.S., \& Van der Linden, D. (2014). Evidence for the general factor of personality as social-effectiveness. Personality and Individual Differences, 64, 147-151.

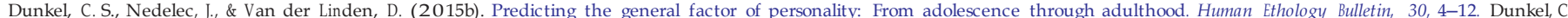
S., Reeve, C. L., Woodley of Menie, M. A., \& van der Linden, D. (2015a). A com- parative study of the general factor of personality in Jewish and non-Jewish populations. Personality and Individual Differences, 78, 63-67.

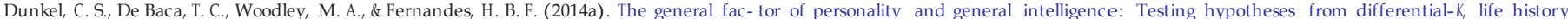
theory, and strategic differentiation-integration effort. Personality and Individual Differences, 61-62, 13-17.

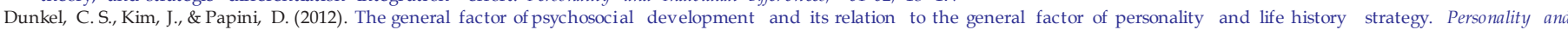
Individual Differences, 52, 202-206.

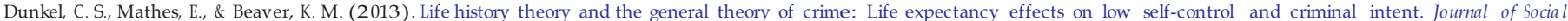
Evolutionary, and Cultural Psychology., 7, 12-23.

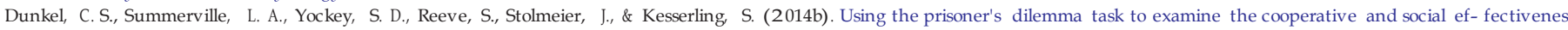
hypotheses of the general factor of personality. Human Ethology Bulletin, 29, 14-22.

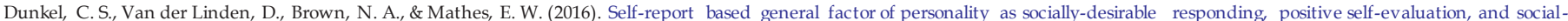
effectiveness. Personality and Individual Differences, 92, 143-147.

Edwards, A. L. (1957). The social desirability variable in personality assessment and research. London: Dryden Press.

Falconer, D. S. (1989). Introduction to quantitative genetics. London: Longman.

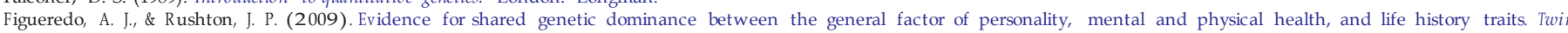
Research and Human Genetics, 12, 555-563.

Figueredo, A.J.,Vásquez, G., Brumbach, B.H.,\& Schneider, S.M.R. (2004). The heritability of life history strategy: The K-factor, covitality, and personality. Social Biology, 51, 121-143.

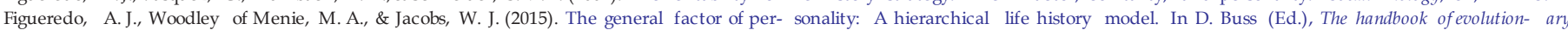
psychology. London: Wiley.

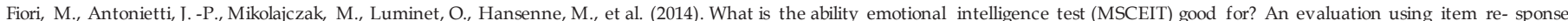
theory. PloS One, 9, e98827. http://dx.doi.org/10.1371/journal.pone.0098827.

He, J., \& van de Vijver, F. J. (2013). A general response style factor: Evidence from a multi-ethnic study in the Netherlands. Personality and Individual Differences, 55, 794-800. 


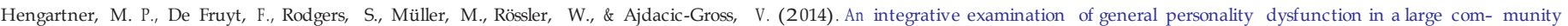
sample. Personality and Mental Health, 8, 276-289.

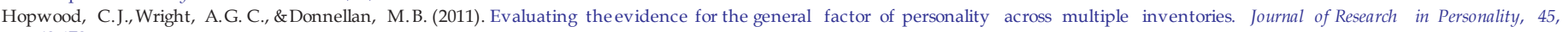
68-478.

Irwing, P. (2013). The general factor of personality: Substance or artefact? Personality and Individual Differences, 55, 534-542.

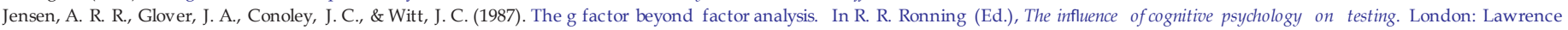
Erlbaum.

Jensen, A. R. (1998). The g factor: The science of mental ability. London: Praeger.

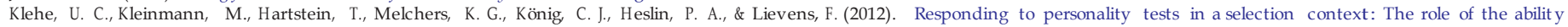
to identify criteria and the ideal-employee factor. Human Performance, 25, 273-302.

Loehlin, J. C. (2012). How general across inventories is a general factor of personality? Journal of Research in Personality, 46, 258-263.

Loehlin, J.C., \& Martin, N. G. (2011). What does a general factor of personality look like in unshared environmental variance? Personality and Individual Differences, 51, 862-865.

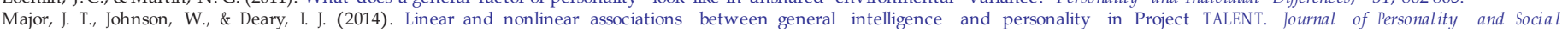
Psychology, 106, 638-654.

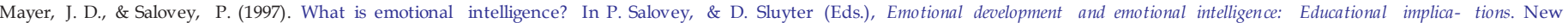
York: Basic Books.

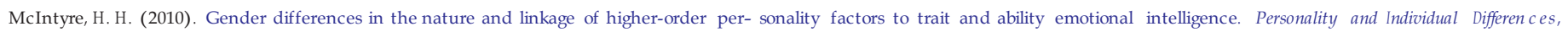
$48,617-622$.

Musek, J. (2007). A general factor of personality: Evidence for the Big One in the five-fac- tor model. Journal of Research in Personality, 41, 1213-1233.

Olderbak, S., Gladden, P., Wolf, P. S. A., \& Figueredo, A. J. (2014). Comparison of life history strategy measures. Personality and Individual Differences, 58, 82-88.

Open Science Collaboration. (2012). An open, large-scale, collaborative effort to estimate the reproducibility of psychological science. Perspectives on Psychological Science, 7, 657-660.

Peabody, D., \& Goldberg, L. R. (1989). Some determinants of factor structures of personal- ity-trait descriptors. Journal of Personality and Social Psychology, 57, 552-567.

Penke, L., Miller, G. F., \& Denissen, J. J. A. (2007). The evolutionary genetics of personality, European Journal of Personality, 21, 549-587.

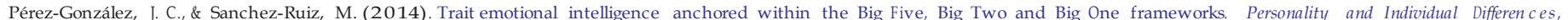
$65,53-58$.

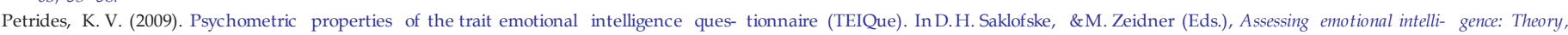
research, \& applications. London: Springer.

Petrides, K. V. (2010). Trait emotional intelligence theory. Industrial and Organizational Psychology: Perspectives on Science and Practice, 3, 136-139.

Petrides, K. V., \& Furnham, A. (2000). On the dimensional structure of emotional intelli- gence. Personality and Individual Differences, 29 , 313-320.

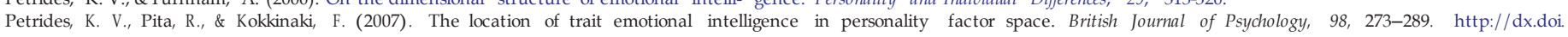
org/10.1348/000712606X120618

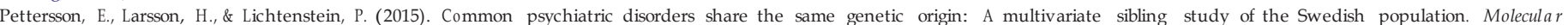
Psychiatry. http://dx.doi.org/10.1038/mp. 2015.116.

Pettersson, E., Turkheimer, E., Horn, E. E., \& Menatti, A. R. (2012). The general factor of personality and evaluation. European Journal of Personality, 26, 292-302.

Revelle, W., \& Wilt, J. (2013). The general factor of personality: A general critique. Journal of Research in Personality, 47, 493-504. 


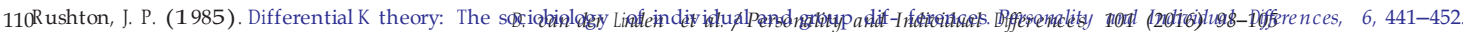

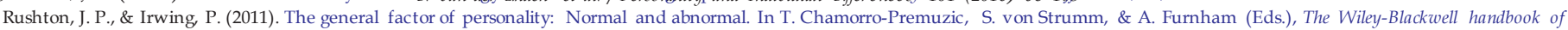
individual differences. London: Blackwell Publishing.

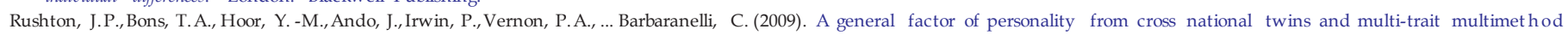
data. Twin Research and Human Genetics, 12, 356-365.

Rushton, J. P., Bons, T. A., \& Hur, Y. (2008). The genetics and evolution of a general factor of personality. Journal of Research in Personality, 42, 1173-1185

Saucier, G. (1994). Separating description and evaluation in the structure of personality attributes. Journal of Personality and Social Psychology, 66, 141-154.

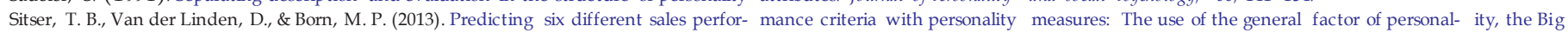
Five and narrow traits. Human Performance, 26, 126-149.

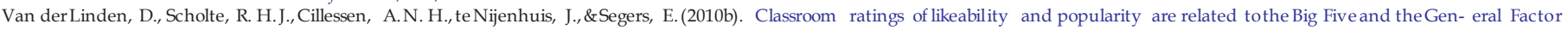
of Personality. Journal of Research in Personality, 44, 669-672.

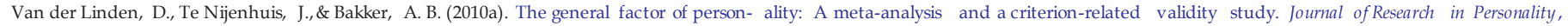
44, 315-327.

Van der Linden, D., Bakker, A. B., \& Serlie, A. (2011b). The general factor of personality in selection and assessment samples. Personality and Individual Differences, 51, 241-245.

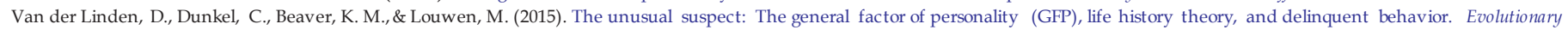
Behavioral Sciences, 9, 145-160.

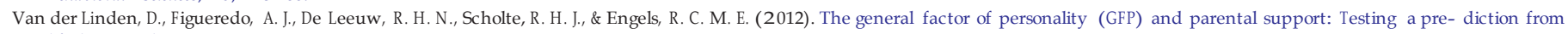
life history theory. Evolution and Human Behavior, 33, 537-546.

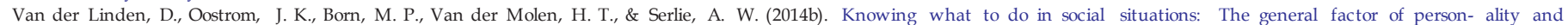
performance on situational judgment tests. Journal of Personnel Psychology, 13, 107-115.

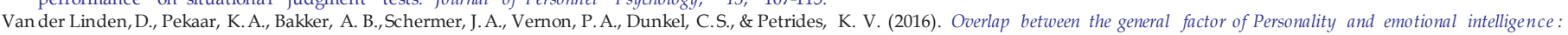
A meta-analysis and a genetic correlation study.

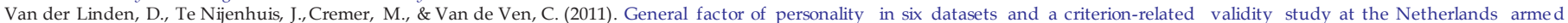
forces. International Journal of Selection and Assessment, 19, 157-169.

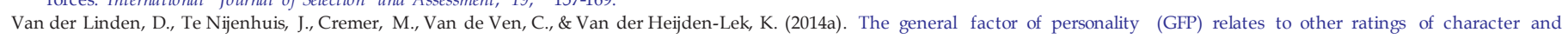
integrity: Two validity studies in personnel selection and training of the Dutch armed forces. International Journal of Selection and Assessment, $22,261-271$.

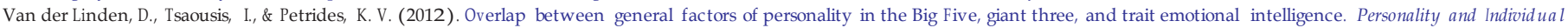
Differences, 53, 175-179.

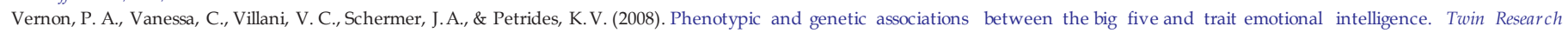
and Human Genetics, 11, 524-530.

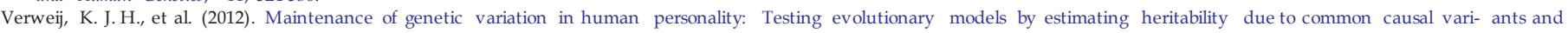
investigating the effect of distant inbreeding. Evolution, 66, 3238-3251.

Veselka, L., Just, C., Jang, K. L.,Johnson, A. M., \&Vernon, P. A. (2012). The general factor of personality: A critical test. Personality and Individual Differences, 52, 261-264.

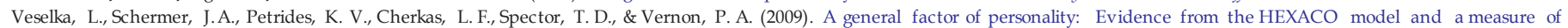
trait emotional intelligence. Twin Research and Human Genetics, 12, 420-424.

Webb, E. (1915). Character and intelligence. An attempt at an exact study of character. Lon- don: Cambridge University Press. 